\title{
Performance Analysis of FinFET based Carry save Adder Cell with Predictive Technology Models
}

\author{
Suresh Singh Baghel \\ Student, M.Tech VLSI Design \\ ACS Division, Centre for \\ Development of Advanced \\ Computing (C-DAC), Mohali, \\ 160071, India
}

\author{
Manjit Kaur \\ Engineer \\ ACS Division, Centre for \\ Development of Advanced \\ Computing (C-DAC), Mohali, \\ 160071, India
}

\author{
Gurmohan Singh \\ Senior Engineer \\ DEC Division, Centre for \\ Development of Advanced \\ Computing (C-DAC), Mohali, \\ 160071, India
}

\begin{abstract}
As scaling of conventional metal-oxide-semiconductor field effect transistor is approaching its fundamental and technological limits, alternate device solutions are being developed. FinFET is rapidly replacing conventional CMOS transistors as it offer lot of improvements in power consumption, propagation delay and propagation delay product (PDP). This paper presents design \& simulation of a double gate FinFET based ultra low power 2-bit Carry Save Adder (CSA) cell. A comprehensive comparison of FinFET and CMOS based 2-bit carry save adder has been performed. The CMOS \& FinFET based 2-bit carry save adder circuits are evaluated at $32 \mathrm{~nm} \& 45 \mathrm{~nm}$ nanoscale technology nodes using Predictive Technology Models (PTM). At 45nm technology node, the FinFET based carry save adder results shows average power consumption reduction of $39.75 \%$; propagation delay reduction of $92.50 \%$ and a propagation delay product (PDP) improvement of $94.42 \%$ as compared to CMOS counterparts. The FinFET based carry save adder results shows average power consumption reduction of 42.19\%; propagation delay reduction of $86.86 \%$ and a propagation delay product (PDP) improvement of $92.22 \%$ as compared to CMOS based carry save adder at $32 \mathrm{~nm}$ technology node.
\end{abstract}

\section{Keywords}

CMOS, FinFET, CSA, PDP, AND, XOR, OR, Double-gate.

\section{INTRODUCTION}

Microprocessor is the important element in the digital circuit design. The arithmetic unit of microprocessor consist of adders as one of the basic element. Among various adders, carry save adder (CSA) has lowest PDP. Nowadays, the major concerns required in the designing of the digital circuits are in the field of power dissipation and propagation delay. PDP is one of the most important performance metric in digital circuit design. As the CMOS devices are scaling day by day, this leads to short channel effects, process reliability degradation and process variation. In order to overcome these problems, new technologies are adopted like FinFET. The FinFET have multi-gate structure which improves mobility, negligible short channel effects, minimum random dopant fluctuations, reduced parasitic junction capacitance and hence improved area efficiency [1-7].

Double Gate FinFET has two gates, one is front gate and other is back gate, it provides flexibility in design with low power and delay. Due to its low leakage structure, the current strength ratio also improves [8-12]. FinFET top and cross- sectional view is demonstrated in fig. 1(a) and (b) respectively.

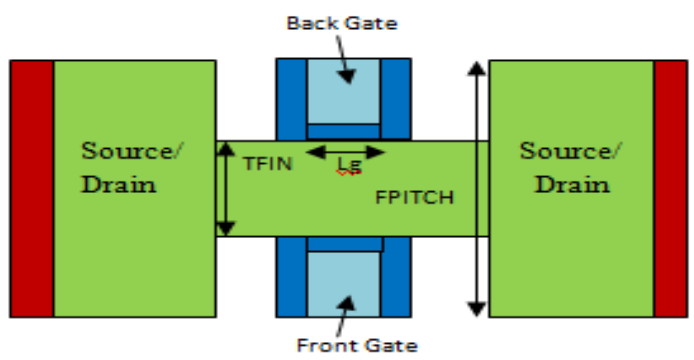

(a)

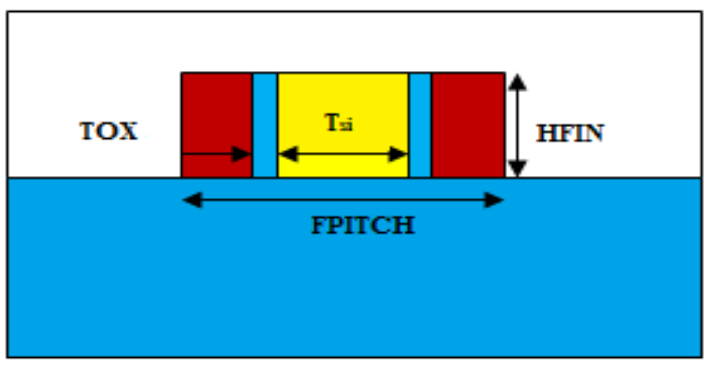

(b)

Fig. 1(a) Top View of FinFET (b) Cross-sectional view of FinFET

Figure 1(b) shows a FinFET multi-fin structure, where $\boldsymbol{T}_{s i}$ represents the thickness of thin silicon body of the fin which is connected to gate electrodes. The channel is upright to the hydroplane of the wafer and current is parallel to hydroplane of the wafer. The front gate and back gate becomes control independent by etching away electrode gate from the top of the channel. The effective gate width of FinFET is given as $2 * \boldsymbol{n} * \boldsymbol{h}$; where, $\mathrm{h}$ is the height and $\mathrm{n}$ is number of fins in the structure.

Doped channel FinFETs are suitable for system on chip (SOC) which need multiple threshold voltages on the same die because back gate of FinFET can be used to adjust threshold voltages. For a non planner device structure, FinFET technology is better replacement in terms of efficiency [13-17].

For predictive modeling, Berkeley Predictive Technology Model (BPTM) was developed for multi-gate transistor 
FinFET using the Berkeley common multi-gate (BSIMMG). HP (high performance) PTM models for $32 \mathrm{~nm} \& 45 \mathrm{~nm}$ technology nodes have been used for CMOS \& MGTMOS (Multi Gate Transistor MOS) [18-20].

\section{CARRY SAVE ADDER DESIGN}

A 2-bit CSA circuit is designed that have low power consumption and delay. The simulation of 2-bit CSA circuit is carried out using H-SPICE simulation tool. The CSA circuit consists of 4 XOR gates and 3 AND gates as shown in the fig. 2. The $\mathrm{a}_{0}, \mathrm{a}_{1}, \mathrm{~b}_{0}$ and $\mathrm{b}_{1}$ are inputs of CSA and $\mathrm{s}_{0}, \mathrm{~s}_{1}$ and $\mathrm{s}_{2}$ are outputs where $s_{0}$ is least significant bit (LSB)) and $s_{2}$ is most significant bit (MSB) and VDD and GND are the power supply rails used in the circuit.

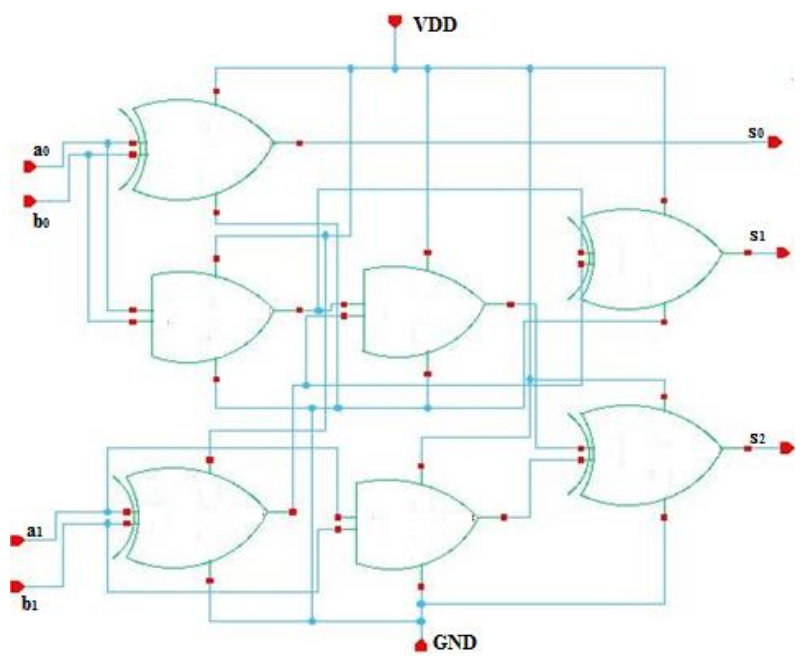

Fig. 2: Two Bit Carry Save Adder

Table 1 is the truth table of designed 2-bit CSA. Various possible input combinations are taken and the output waveform is shown in fig. 3 .

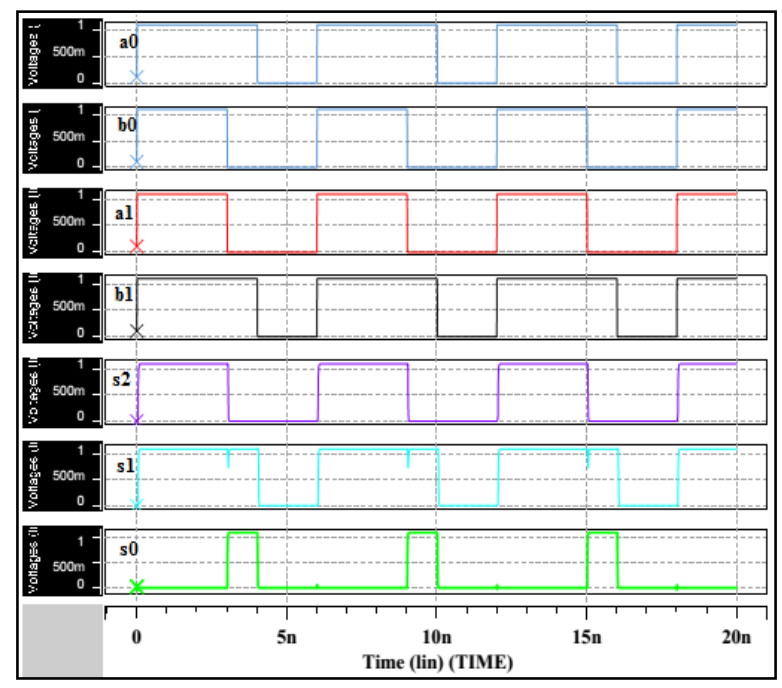

Fig. 3 Output waveform of 2-bit CSA

\subsection{CMOS Based Carry Save Adder}

CSA has basically two building blocks i.e. XOR and AND gates. The XOR gate is used to get SUM as output in adder circuit. XOR circuit consists of 8 NMOS and 8 PMOS transistors as shown in the fig. 4. Similarly AND gate is used to get carry as output. It consists of 3 PMOS and 3 NMOS transistors as shown in Fig. 5.
Table 1. 2-bit Carry save adder truth table

\begin{tabular}{ccccccc}
\hline & \multicolumn{2}{c}{ Inputs } & & \multicolumn{3}{c}{ Output } \\
a0 & $\mathbf{b 0}$ & $\mathbf{a 1}$ & $\mathbf{b 1}$ & $\mathbf{s 0}$ & $\mathbf{s 1}$ & $\mathbf{s 2}$ \\
\hline 0 & 0 & 0 & 0 & 0 & 0 & 0 \\
0 & 0 & 0 & 1 & 0 & 1 & 0 \\
0 & 0 & 1 & 0 & 0 & 1 & 0 \\
0 & 0 & 1 & 1 & 0 & 0 & 1 \\
0 & 1 & 0 & 0 & 0 & 0 & 1 \\
0 & 1 & 0 & 1 & 1 & 1 & 0 \\
0 & 1 & 1 & 0 & 1 & 1 & 0 \\
0 & 1 & 1 & 1 & 1 & 0 & 1 \\
1 & 0 & 0 & 0 & 1 & 0 & 0 \\
1 & 0 & 0 & 1 & 1 & 1 & 0 \\
1 & 0 & 1 & 0 & 1 & 1 & 0 \\
1 & 0 & 1 & 1 & 1 & 0 & 1 \\
1 & 1 & 0 & 0 & 0 & 1 & 0 \\
1 & 1 & 0 & 1 & 0 & 0 & 1 \\
1 & 1 & 1 & 0 & 0 & 0 & 1 \\
1 & 1 & 1 & 1 & 0 & 1 & 1 \\
\hline & & & & & &
\end{tabular}

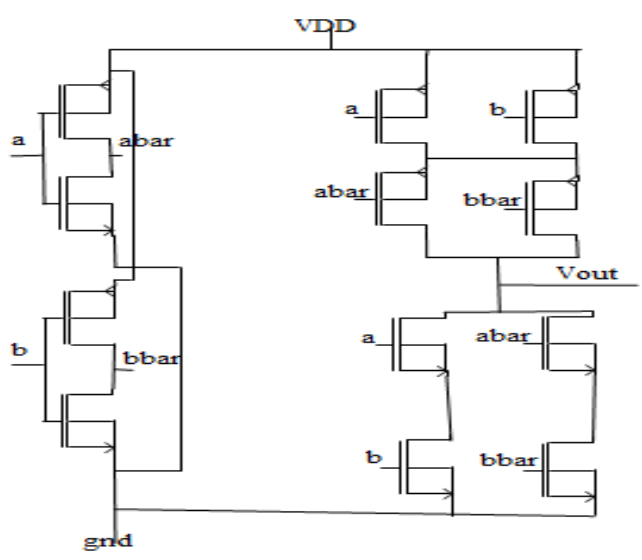

Fig. 4 CMOS based XOR gate

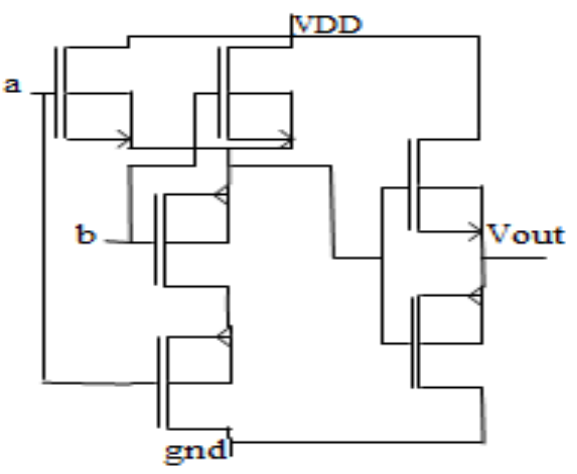

Fig. 5 CMOS based AND gate 


\subsection{FinFET Based Carry Save Adder}

Double Gate (DG) FinFET transistors are used to design 2-bit CSA cell. The front and back gate of DG FinFET has self determining control which can be efficiently used to design high performance and low power circuits. For selfdetermining gate control, front and back transistors are connected. DG FinFET have back gate which makes it different from conventional CMOS transitors, hence reduces leakage current and short channel effects. There are two modes of operation in FinFET viz. Independent Gate (IG) and Short Gate (SG). In the IG FinFETmode, front gate and back gate is connected by different signal, it has five terminalsfront gate, back gate, drain, source and bulk. In the SG FinFET front gate and back gate are connected together hence it has four terminals-gate, drain, source and bulk similar to conventional CMOS transistor. The FinFET based schematic design of 2-bit XOR gate and AND gates are shown in Fig. 6 and 7 respectively.

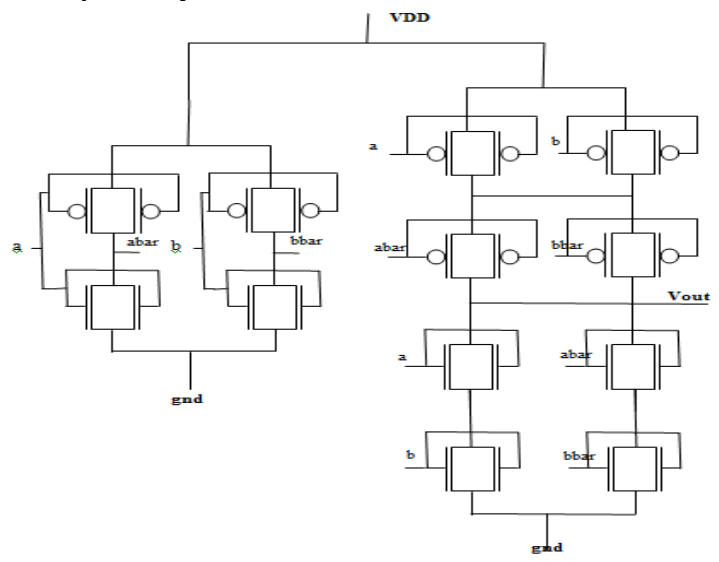

Fig. 6 Schematic design of FinFET based XOR gate

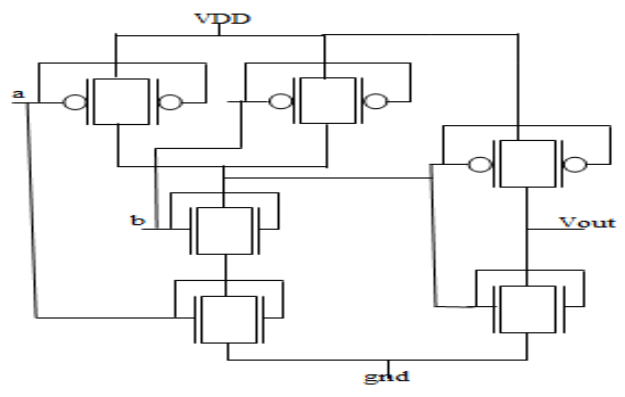

Fig. 7 Schematic design of FinFET based AND gate

\section{RESULTS \& DISCUSSIONS}

\subsection{Simulation results of basic N-type Transistor}

The table 2 shows comparison of n-type transistor for CMOS and FinFET technologies at $45 \mathrm{~nm}$ and $32 \mathrm{~nm}$ nodes.

Table 2. Performance comparison of CMOS and FinFET based n-type transistor at $45 \mathrm{~nm}$ and $32 \mathrm{~nm}$ node

\begin{tabular}{lcccc}
$\begin{array}{l}\text { N-Type } \\
\text { Transistor } \\
\text { Parameter }\end{array}$ & $\begin{array}{c}45 \mathrm{~nm} \\
\text { CMOS }\end{array}$ & $\begin{array}{c}45 \mathrm{~nm} \\
\text { FinFET }\end{array}$ & $\begin{array}{c}32 \mathrm{~nm} \\
\text { CMOS }\end{array}$ & $\begin{array}{c}32 \mathrm{~nm} \\
\text { FinFET }\end{array}$ \\
\hline Length(nm) & 50 & 50 & 40 & 40 \\
Width(nm) & 200 & 200 & 160 & 160 \\
$\mathrm{~V}_{\mathrm{dd}}(\mathrm{volt})$ & 1.1 & 1.1 & 0.9 & 0.9 \\
$\mathrm{I}_{\mathrm{ds}}(\mu \mathrm{A})$ & 275 & 525 & 170 & 310 \\
\hline
\end{tabular}

Maximum drain current in n-type FinFET transistor is $525 \mu \mathrm{A}$, while it is $275 \mu \mathrm{A}$ in conventional n-type CMOS at $45 \mathrm{~nm}$ technology node i.e. n-type FinFET transistor provides $47 \%$ more drain current. At $32 \mathrm{~nm}$ technology node, maximum drain current in n-type FinFET transistor is $310 \mu \mathrm{A}$, while it is $170 \mu \mathrm{A}$ in conventional n-type CMOS i.e. n-type FinFET transistor provides $42 \%$ more drain current. Supply voltage $\left(\mathrm{V}_{\mathrm{dd}}\right)$ is $1.1 \mathrm{~V}$ for $45 \mathrm{~nm}$ node and $0.9 \mathrm{~V}$ for $32 \mathrm{~nm}$ node. The bar graphs in fig. 8 shows the comparison of maximum drain current at $45 \mathrm{~nm}$ and $32 \mathrm{~nm}$ technologies for n-type FinFET and the CMOS transistors.

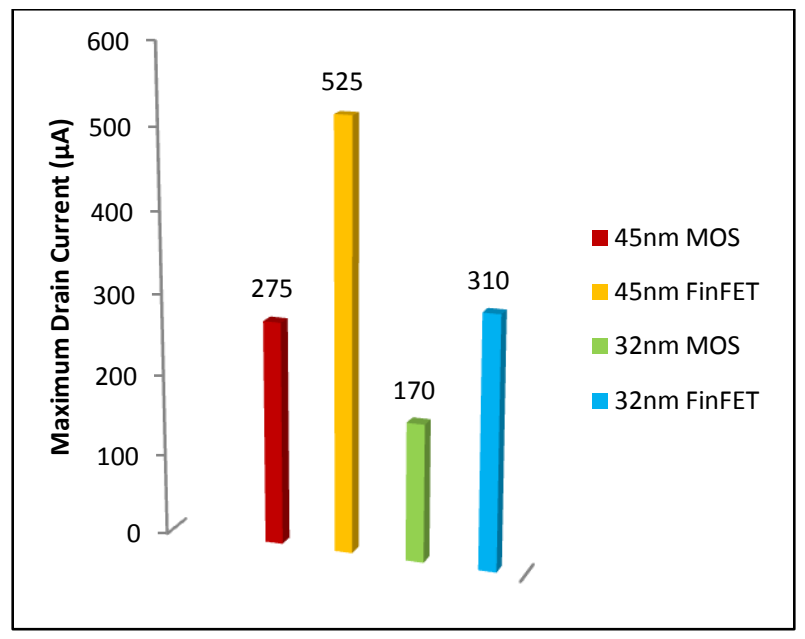

Fig. 8 Comparison of maximum drain current for CMOS \& FinFET n-type transistors at $45 \mathrm{~nm}$ and $32 \mathrm{~nm}$ technologies

\subsection{Carry Save Adder}

The table 3 lists simulation results for CMOS \& FinFET based carry save adder results at $45 \mathrm{~nm}$ and $32 \mathrm{~nm}$ technology nodes respectively. It is observed that there is $40.07 \%$ \& $42.19 \%$ reduction in average power in FinFET based carry save adder as compared to conventional CMOS based carry save adder at $45 \mathrm{~nm} \& 32 \mathrm{~nm}$ technology nodes respectively. Propagation delay reduction is $92.5 \%$ \& $86.55 \%$ and powerdelay product improvement is $94.40 \%$ \& $91.79 \%$ in FinFET based carry save adder at $45 \mathrm{~nm} \& 32 \mathrm{~nm}$ technology nodes. Fig. 9, 10 and 11 shows the bar-graph comparison of average power, propagation delay \& power-delay product (PDP) at $45 \mathrm{~nm}$ and $32 \mathrm{~nm}$ nodes in the CMOS \& FinFET technologies based CSA respectively.

Table 3. Performance comparison of CMOS and FinFET based 2-bit Carry Save Adders at $45 \mathrm{~nm}$ and $32 \mathrm{~nm}$ node

\begin{tabular}{lcccc}
\hline Parameter & CMOS & FinFET & CMOS & FinFET \\
& $\mathbf{( 4 5 n m )}$ & $\mathbf{( 4 5 n m )}$ & $\mathbf{( 3 2 n m )}$ & $\mathbf{( 3 2 n m )}$ \\
\hline $\begin{array}{l}\text { Avg. } \\
\text { Power( } \boldsymbol{\mu W})\end{array}$ & 24.36 & 14.60 & 14.09 & 8.145 \\
$\begin{array}{l}\text { Propagation } \\
\text { Delay(ns) }\end{array}$ & 0.520 & 0.039 & 0.119 & 0.016 \\
$\begin{array}{l}\text { PDP(fJ) } \\
\text { (12.67 }\end{array}$ & 0.582 & 1.670 & 0.137 \\
\hline
\end{tabular}




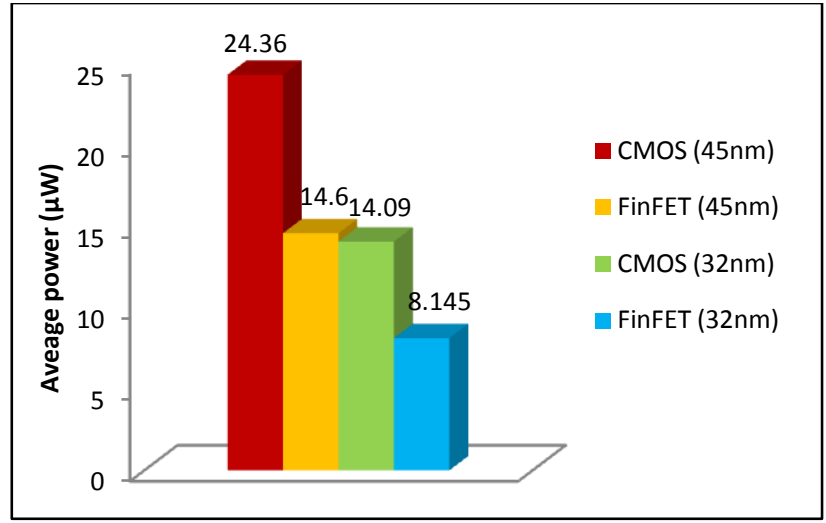

Fig. 9 Comparison of average power of FinFET and CMOS based CSA at $32 \mathrm{~nm} \& 45 \mathrm{~nm}$ technology nodes

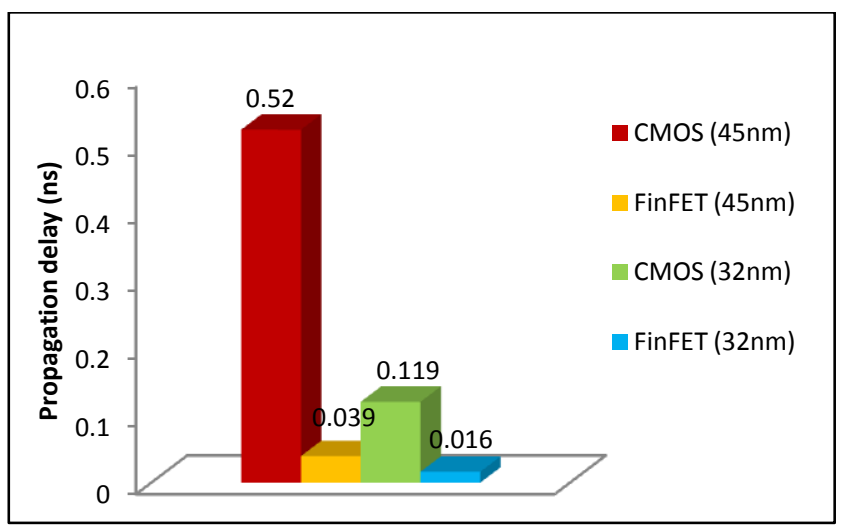

Fig. 10 Comparison of propagation delay of FinFET and CMOS based CSA at 32nm \& $\mathbf{4 5} \mathrm{nm}$ technology nodes

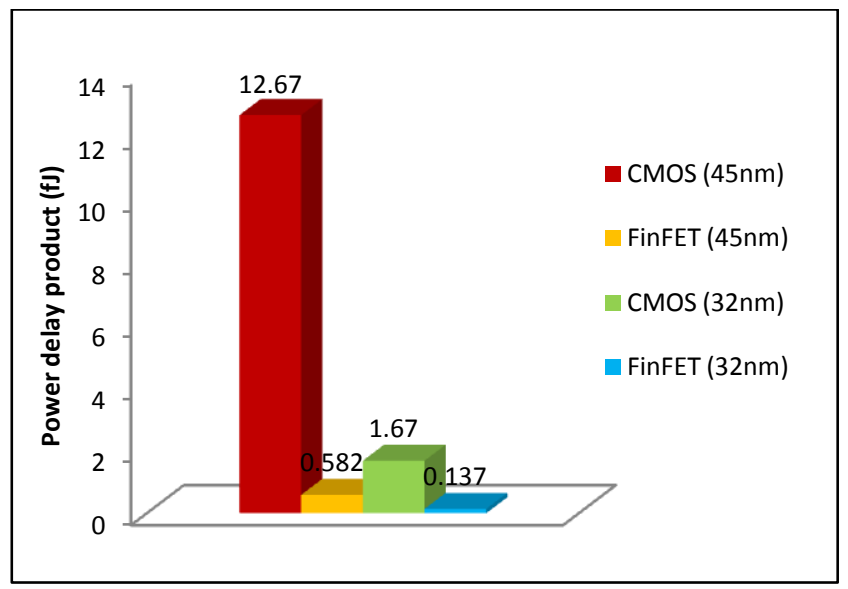

Fig. 11 Comparison of power-delay product of FinFET and CMOS based CSA at $32 \mathrm{~nm} \& 45 \mathrm{~nm}$ technology nodes

\section{CONCLUSION}

As the FinFET offers lower leakage current and power dissipation; it became most favorable device beyond $22 \mathrm{~nm}$ technology node. As an emerging new technology, FinFET offers many dimensions where research work can be explored. So, a carry save adder is designed using FinFET transistors and carried out its comparison with its CMOS counterpart. In this research work, the comparative analysis of circuits based on FinFET and CMOS technologies at $45 \mathrm{~nm}$ and $32 \mathrm{~nm}$ has been performed using H-SPICE tool. The predictive technology models (PTM) are used for simulation \& analysis of CMOS and FinFET based 2-bit carry save adder circuits at $32 \mathrm{~nm} \& 45 \mathrm{~nm}$ nanoscale technology nodes. FinFET based carry save adder results shows average power consumption reduction of $39.75 \%$ and $42.19 \%$; propagation delay reduction of $92.50 \%$ and $86.86 \%$ and a propagation delay product (PDP) improvement of $94.42 \%$ and $92.22 \%$ as compared to CMOS counterparts at $45 \mathrm{~nm}$ and $32 \mathrm{~nm}$ technologies respectively. The results verified that FinFET technology outperforms CMOS technology in terms of power consumption, delay and power-delay product (PDP).

\section{REFERENCES}

[1] D. Hisamoto, W.-C. Lee, J. Kedzierski et al., "FinFETa self aligned double-gate MOSFET scalable to $20 \mathrm{~nm}$," IEEE Transactions on Electron Devices, vol.47, no.12, pp. 2320-2325, 2000.

[2] B. Raj, A. K. Saxena and S. Dasgupta, "Quantum Mechanical Analytical Modeling of Nanoscale DG FinFET: Evaluation of Potential, Threshold Voltage and Source/Drain Resistance " Elsevier's Journal of Material Science in Semiconductor Processing, Elsevier, Vol. 16, issue 4, pp. 1131- 1137, 2013.

[3] B. Raj, A. K. Saxena and S. Dasgupta, "Analytical Modeling for the Estimation of Leakage Current and Subthreshold Swing Factor of Nanoscale Double Gate FinFET Device" Microelectronics International, UK, vol. 26, pp. 53-63, 2009

[4] B. Raj, A. K. Saxena and S. Dasgupta, "A Compact Drain Current and Threshold Voltage Quantum Mechanical Analytical Modeling for FinFETs" Journal of Nanoelectronics and Optoelectronics (JNO), USA, vol. 3, no. 2, pp. 163-170, 2008.

[5] B. Yu, L. Chang, S. Ahmed et al., "FinFET scaling to 10 nm gate length," in Proceedings of the IEEE International Devices Meeting (IEDM '02), pp. 251-254, San Francisco, Calif, USA, December 2002.

[6] B. Raj, A. K. Saxena and S. Dasgupta, "Nanoscale FinFET Based SRAM Cell Design: Analysis of Performance metric, Process variation, Underlapped FinFET and Temperature effect" IEEE Circuits and System Magazine, vol. 11, issue 2, pp. 38- 50, 2011.

[7] S. Tang, L. Chang, N. Lindert et al., "FinFET - a quasiplanar double-gate MOSFET," in Proceedings of the International of Solid-State Circuits Conference, pp. 118-119, February 2001.

[8] I.P.Colinge, FinFETs and Other Multi-Gate Transistors, 1st ed. Springer: New York, 2008, pp. 4-25.

[9] K.Kim at al., "Leakage power analysis of 25-nm doublegate CMOS devices and circuits," IEEE Trans. Electron Devices, vol. 52, no. 5, pp.980-986, May 2005.

[10] P. Mishra and N.K. Jha, "Low-power FinFET circuit synthesis using surface orientation optimization," in Proc. Design Automation and Test in Europe, pp. 311-314 March 2010.

[11] K. Akarvardar, C. D. Young, D. Veksler, K.-W. Ang, I. Ok, M. Rodgers et al., "Performance and variability in multi-VT FinFETs using fin doping," in Proc. VLSI TSA, 2012 ,

[12] M. Guillorn, J. Chang, A. Bryant et al., "FinFET performance advantage at $22 \mathrm{~nm}$ : an AC perspective," in Proceedings of the Symposium on VLSI Technology 
Digest of Technical Papers (VLSIT '08), pp.12-13, June2008.

[13] J.Gu, J.Keane, S.Sapatnekar, andC.H.Kim, "Statistical leakage estimation of double gate FinFET devices considering the width quantization property, " IEEE Transactions on Very Large Scale Integration Systems, vol. 16, no. 2, pp. 206-209, 2008.

[14] D.Ha, H.Takeuchi, Y.-K.Choi, and T.-J.King, "Molybdenum gate technology for ultrathin-body MOSFETs and FinFETs, " IEEE Transactions on Electron Devices, vol.51, no.12, pp.1989-1996, 2004.

[15] T. Sairam, W. Zhao, and Y. Cao, "Optimizing FinFET technology for high-speed and low-power design," in Proceedings of the 17th Great Lakes Symposium on VLSI (GLSVLSI '07), pp.73-77, March 2007.

[16] Keunwoo Kim; Kanj, R.; Joshi, R.V., "Impact of FinFET technology for power gating in nano-scale design," Quality Electronic Design (ISQED), 2014 15th International Symposium on, vol., no., pp.543, 547, 3-5 March 2014.

[17] E. R. Hsieh and S. S. Chung, "The proximity of strain induced effect to improve the electron mobility in a silicon carbon source-drain structure of n-channel metaloxide semiconductor field-effect transistors," Appl. Phys. Lett., vol. 96, no. 9, pp. 093501, March 2010.

[18] 32nm sub-circuit model for double gate FinFET[Online] Available: http://ptm.asu.edu/modelcard/32nm_finfet.rar

[19] 45nm sub-circuit model for double gate FinFET[Online]. Available: http://ptm.asu.edu/modelcard/45nm_finfet.rar

[20] S.Sinha et al., "Exploring sub-20nm FinFET design with Predictive Technology Models," in Design Automation Conference (DAC), $201249^{\text {th }} A C M / E D A C / I E E E$, pp. 283 -288 , June 2012. 